\title{
DESSENSIBILIZAÇÃO QUÍMICA, NEURECTOMIA PALMAR E COMPLICAÇÕES PÓS-CIRURGICAS EM EQUINO: RELATO DE CASO
}

[Chemical desensitization, palmar neurectomy and post-surgical complications in horse: case report]

\author{
Natanni C. Vitor Rocha ${ }^{1}$, Pierre Barnabé Escodro ${ }^{1 *}$, Jackellyne Laís Ferreira Lins ${ }^{1}$, Yane Fernandes \\ Moreira $^{1}$, Kezia dos Santos Carvalho², Fernando Wiecheteck Souza ${ }^{1}$ \\ ${ }^{1}$ Grupo de Pesquisa e Extensão em Equídeos da Universidade Federal de Alagoas- GRUPEQUI-UFAL, Viçosa-AL, Brasil. \\ ${ }^{2}$ Curso de Medicina Veterinária CESMAC, Marechal Deodoro-AL, Brasil.
}

\begin{abstract}
RESUMO - Objetiva-se relatar o caso de um garanhão da raça Quarto de Milha de 12 anos, portador de síndrome degenerativa crônica podal e anquilose parcial na articulação metacarpo-falângica do membro torácico esquerdo, submetido à neurólise química palmar com cloreto de amônio $2 \%$, exteriorizando eficácia analgésica e retorno à atividade esportiva por 40 dias. Após o período, foi realizado neurectomia palmar (NP), sendo que com 110 dias o equino apresentou neuromas dolorosos, sendo submetido à nova cirurgia. Há necessidade de mais relatos e pesquisas envolvendo a NP em equino avaliando: tempo e qualidade de analgesia; complicações após NP; reais riscos proprioceptivos ao animal e avaliação de fatores de risco na formação de neuromas.
\end{abstract}

Palavras-Chave: Quarto de Milha; neurólise; cloreto de amônio; neuroma doloroso.

\begin{abstract}
The aim is report the case of a stallion Quarter Horse with 12 years old, with podal chronic degenerative syndrome and partial ankylosis in metacarpal-phalangeal joint of the left forelimb, submitted to palmar chemical neurolysis with ammonium chloride $2 \%$, externalizing analgesic efficacy and return to sports activity for 40 days. After the period, was performed palmar neurectomy (NP), and with 110 days the horse had painful neuromas, underwent the new surgery. It is necessary to more reports and research involving the NP in equine evaluating: time and quality of analgesia; complications after NP; proprioceptive real risks to the animal and assessment of risk factors in the formation of neuromas.
\end{abstract}

Keywords: Quarter Horse; neurolysis; ammonium chloride; painful neuroma.

\footnotetext{
* Autor para correspondência. E-mail: pierre.vet@gmail.com Recebido: 30 de dezembro de 2015.

Aceito para publicação: 12 de fevereiro de 2016.
} 


\section{INTRODUÇÃO}

A terapia da dor crônica compõe grande desafio à Medicina Veterinária. Nos casos crônicos de equinos refratários às terapias convencionais, como na "dor crônica de talão" em equinos (associada às afecções degenerativas podais), pode-se optar de forma paliativa pela interrupção na transmissão nociceptiva, através da neurectomia digital palmar (NDP) ou utilização de fármacos neurolíticos, apresentando como principais complicações o surgimento de neuromas dolorosos, dessensibilização incompleta, tempo insuficiente de ação neurolítica química e reinervações (Dabareiner et al., 1997; Nicoletti et al., 2007; Koch, 2011; Rezaie et al., 2011).

Objetivando a NDP, algumas técnicas são mais comumente utilizadas, entre elas a técnica da guilhotina (TG), a técnica da secção nervosa e cobertura epineural (TCE) e a técnica de stripping ou pull-through (TS) (Black, 1992; Dabareiner et al., 1997; Faleiros et al., 2008; Rezaie et al., 2011). Das técnicas citadas, Faleiros et al. (2008) relatam que TG e TS foram satisfatórias, sendo a TG é mais simples de ser executada, enquanto que a TS apresentou menor potencial para produzir reinervação e neuromas dolorosos por retirar um segmento nervoso até três vezes maior. No entanto, Escobar et al. (2008) concluíram que o retorno da sensibilidade nervosa deve demorar mais a ocorrer após a TS, contudo a maior proporção de tecido nervoso no tecido cicatricial sugere que o uso dessa técnica favorece a regeneração nervosa. Já Rezaie et al. (2011) avaliaram, através de exames histopatológicos, que a TG ocasiona menor índices de neuromas dolorosos em relação a TCE.

Entre as indicações do uso de agentes neurolíticos na medicina equina estão enfermidades onde a neurectomia digital palmar é opção terapêutica. Assim, é cada vez mais comum a utilização destes agentes químicos, tais quais os sais de amônio, fenóis e alcoóis, na rotina clínico-cirúrgica associada aos dígitos, mesmo com discussões acerca da ética envolvida no procedimento, legislações vigentes e riscos proprioceptivos para o conjunto cavalo-cavaleiro (Nicoletti et al, 2007; Escodro et al., 2011; Escodro et al., 2015).

Poucos relatos de neurólise química ou neurectomia palmar (NP) são encontrados na literatura, devido a localização proximal nervosa e risco proprioceptivo ao animal, porém Nicoletti et al. (2007) não notaram diferenças de propriocepção em animais submetidos a neurólise química palmar com álcool etílico absoluto. Escodro et al. (2012) relatam positivamente a NP associada à infiltração de composição etanólica intra-articular para tratamento de osteoartrite interfalângica proximal em equino.
Além disso, Guasco et al. (2013) relatam a neurectomia do ramo profundo lateral nervo palmar como tratamento eficaz na desmite proximal crônica do suspensório do boleto. Este trabalho objetiva relatar o caso de um equino da raça Quarto de Milha submetido à neurólise química com cloreto de amônio a $2 \%$ no NP, retorno a sensibilidade e posterior neurectomia palmar bilateral.

\section{RELATO DE CASO}

Um garanhão da raça Quarto de Milha, 12 anos, pesando $460 \mathrm{~kg}$, de atividade de vaquejada, foi encaminhado ao Ambulatório do Grupo de Pesquisa e Extensão em Equídeos da Universidade Federal de Alagoas (GRUPEQUI-UFAL). Durante a anamnese, obtida através do médico veterinário do animal, foi relatado que o mesmo estava sem participar de competições há mais de um ano, devido a claudicações no membro torácico esquerdo (MTE). Ao exame clínico, observou-se claudicação grau 2 (Stashak, 1994) no MTE, com sensibilidade positiva em sola e talão dos cascos, além de flexão com resistência e positiva para a dor na articulação metacarpo-falângica (após flexão aumentava para claudicação grau 3). Foi efetuado o bloqueio anestésico dos nervos digitais palmares com $5 \mathrm{~mL}$ de lidocaína a $2 \%$ sem vasoconstritor em cada ramo nervoso do MTE, não diminuindo significativamente o grau de claudicação, porém a mesma cessou após a anestesia perineural dos nervos palmares (NP). O animal não apresentou déficit de locomoção visível ao trote e galope após o bloqueio.

No exame radiológico, na incidência dorso-palmar, o MTE apresentava lesões sugestivas de síndrome degenerativa crônica podal (com comprometimento de sola), além de diminuição de espaço articular e presença de osteófitos na articulação metacarpo falângica, sugerindo início de anquilose articular. Os achados radiológicos corroboram com o fato da não efetividade do bloqueio perineural digital palmar para cessamento da claudicação (Figura 1).

O tratamento instaurado foi a infiltração com hialuronato de sódio $(20 \mathrm{mg})$ e triancinolona (18 mg) na articulação metacarpo falângica esquerda e infiltração perineural nos dois nervos palmares (laterais e mediais) do MTE, com cloreto de amônio a $2 \%$, sendo $5 \mathrm{~mL}$ em cada ramo nervoso. Após 10 dias o cavalo foi reavaliado apresentando ausência de sensibilidade no casco, sendo liberado para início de treinamento. Aos 40 dias, após participação em duas provas, a sensibilidade retornou no MTE, momento que optou-se pela NP, com recomendação do animal só ser utilizado para montaria e reprodução. 


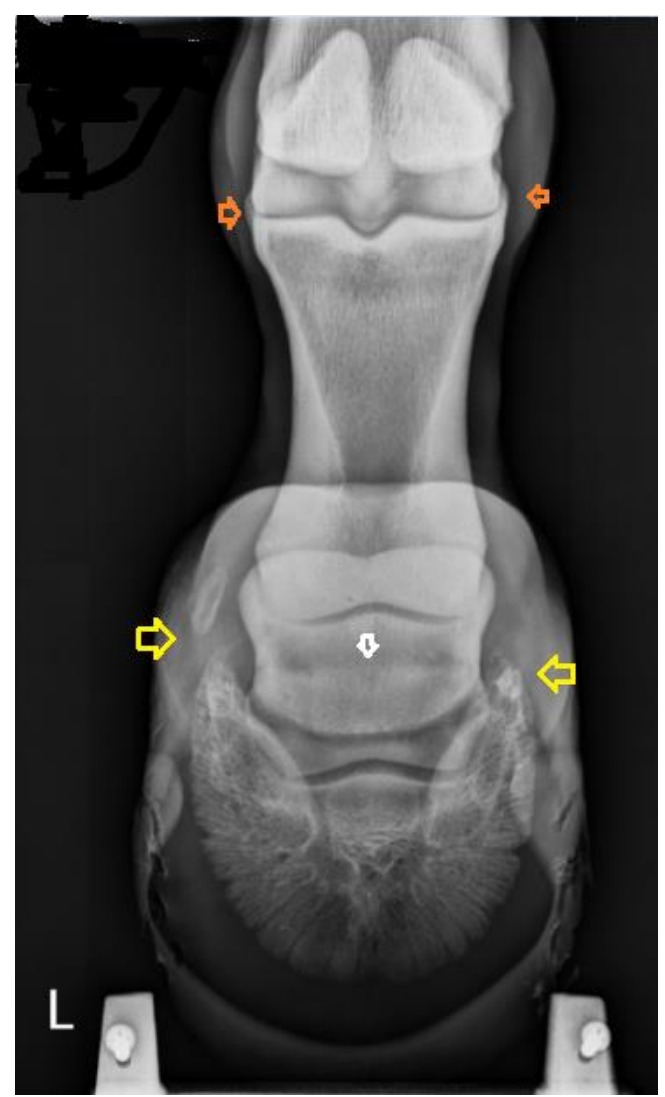

Figura 1. Incidência dorso-palmar do MTE: Setas vermelhas são sugestivas de osteófitos na articulação metacarpo-falângica (em concomitância com a dor e resistência a flexão no exame clínico sugere anquilose parcial). Setas amarelas indicam fratura da ossificação de cartilagem alar lateral e reação periostal na medial; Seta branca indica perda no limite cortico-medular do osso sesamóide distal, além do casco apresentar dilatação de canais vasculares.
Após condutas pré-cirurgicas de rotina, o animal foi pré-medicado com xilazina $(0,5 \mathrm{mg} / \mathrm{kg}, \mathrm{IV})$ e, após 20 minutos, submetido anestesia intravenosa total (TIVA): indução com Diazepan $(0,1 \mathrm{mg} / \mathrm{kg})$ e cloridrato de ketamina, $(2 \mathrm{mg} / \mathrm{kg})$; manutenção com éter gliceril guaiacol, na dose de $50 \mathrm{mg} / \mathrm{kg}$, diluído em $500 \mathrm{~mL}$ de solução fisiológica, com diluição de $800 \mathrm{mg}$ de ketamina e $400 \mathrm{mg}$ de xilazina na solução. A NP adaptada da TG em nervos digitais palmares foi realizada com o animal em decúbito lateral. Foram retirados segmentos nervosos com média de $4,5 \mathrm{~cm}$, após minuciosa dissecação devido a aderência nervosa na artéria palmar presente nos quatro campos cirúrgicos. Ao final da cirurgia, foram aplicadas bandagens de Robert Jones sobre os membros, as quais foram substituídas a cada três dias até a retirada dos pontos. O animal ficou internado durante 15 dias, e recebeu alta médica com a indicação de repouso por 60 dias e aplicação intramuscular de $5 \mathrm{~mL}$ de sulfato de condroitina a $12 \%$ a cada 7 dias, durante 4 meses.

Mesmo com a indicação de não participação em provas, o paciente retornou as atividades de vaquejada, porém foi reencaminhado para o GRUPEQUI-UFAL após 110 dias, onde constatouse massa dolorosa nos cotos nervosos nas duas faces do MTE, sendo identificados neuromas dolorosos. $\mathrm{O}$ animal foi submetido à nova cirurgia para exéreses de neuromas, com retirada de massa amorfas com larguras de 1,3 (lateral) e 1,2 (medial) $\mathrm{cm}$; e comprimento de 6 (lateral) e $6,2 \mathrm{~cm}$ de comprimento (medial), sendo indicado afastamento da carreira esportiva (Figura 2). Após a exérese dos neuromas, o animal foi acompanhado até 120 dias, sem nova recidiva, porém ainda em repouso absoluto, só sendo utilizado para reprodução.

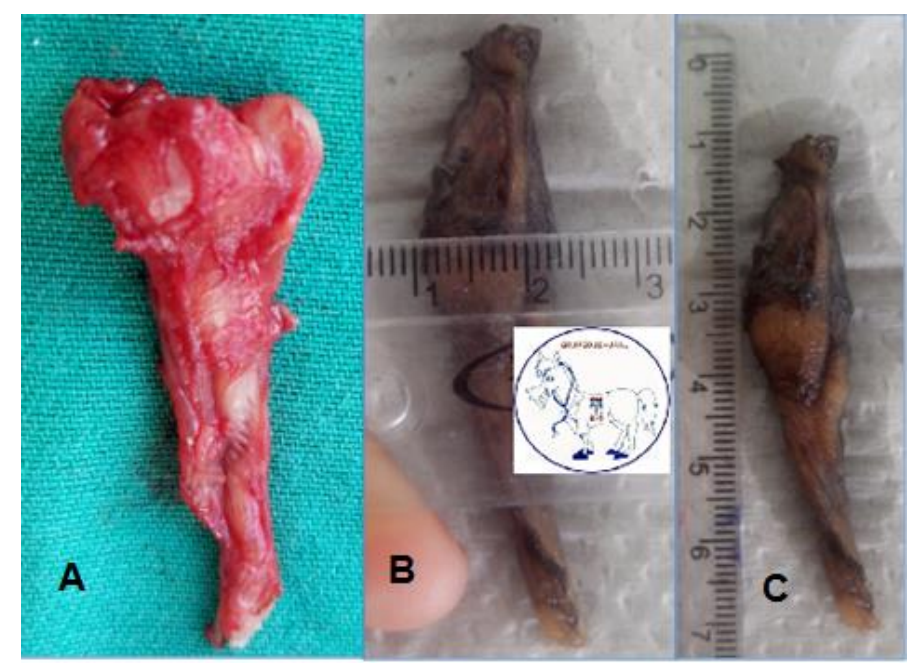

Figura 2. Neuroma retirado no Nervo Palmar lateral do MTE: A- Imagem logo após exérese; Mensuração no neuroma no momento anterior ao exame histopatológico: Largura- 1,3 com (B) e Comprimento- 6,0 cm (C). 
Ao exame histopatológico observou-se proliferação de tecido conjuntivo associado a neovascularização, caracterizando tecido cicatricial. Os feixes de fibras nervosas apresentaram diminuição da quantidade de fibras axonais degeneradas e vacuolizadas.
Observou-se ainda ausência de perineuro, em algumas áreas, associadas à proliferação de fibras conjuntivas ao redor de feixes neuronais (Figura 3) achados compatíveis aos encontrados por Rezaie et al. (2011) em nervos digitais palmares.

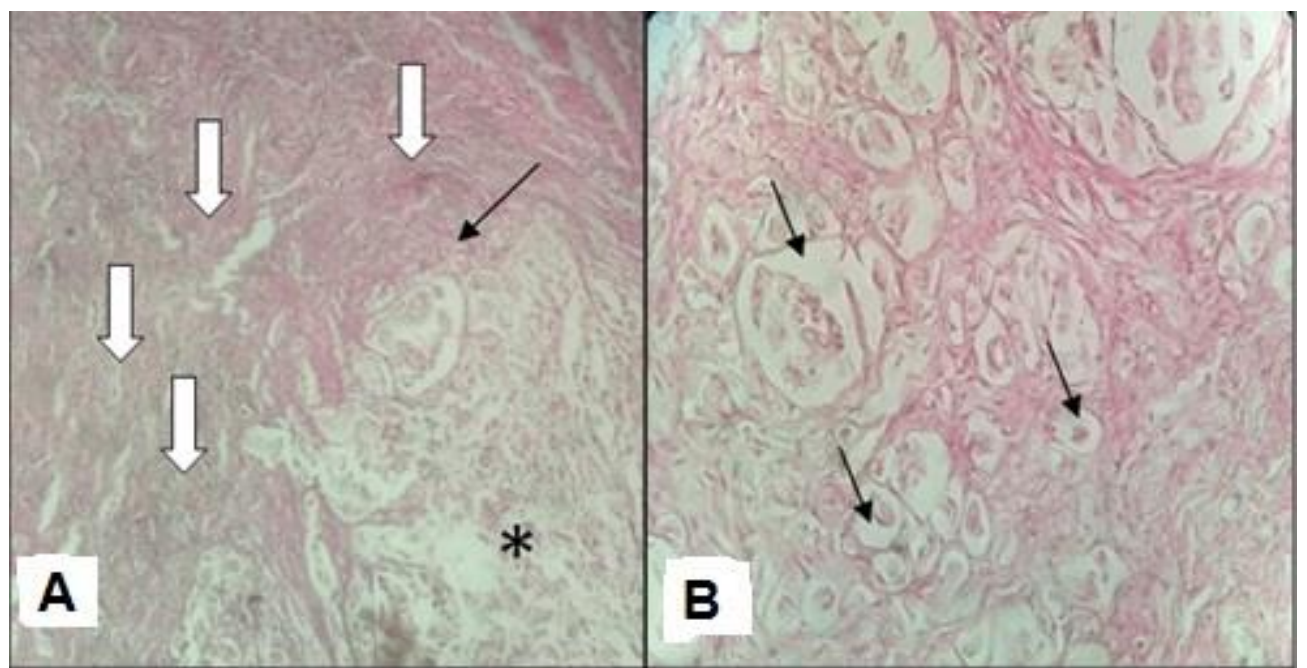

Figura 3. Imagem Microscópica (HE) do neuroma palmar lateral, obj de 10 X: A: Observa-se proliferação de tecido fibroso cicatricial ao redor de feixe nervoso (setas brancas), ausência de perineuro (seta preta) associado a degeneração e vacuolização de feixe neuronal (*).B: Observa-se diferentes graus de vacuolização no interior degeneração de fibra nervosa (seta apontando para vacuolização).

\section{DISCUSSÃO}

No relato de caso, a neurólise química palmar através da solução de cloreto de amônio $2 \%$ perineural foi eficaz por cerca de 40 dias, sugerindo lesões nervosas de neuropaxia (perda da condução dos impulsos nervosos, porém sem degeneração estrutural do nervo) ou axoniotimese (nervo com preservação de epineuro, perineuro e células de Schwann), ambas permitindo a regeneração da função nervosa de semanas e meses, conforme já relatado por Nicoletti et al. (2007) em infiltrações perineurais palmares com extrato de planta "pitcher" em álcool benzílico 0,75\%. Ainda, é relevante afirmar que o bloqueio neurolítico ou neurectomia palmar só é indicado se o animal não apresentar déficit proprioceptivo visível ao bloqueio anestésico prévio e analgesia satisfatória (Escodro et al., 2011), como no caso descrito.

Apesar da neurectomia digital palmar ser utilizada há décadas como forma de aliviar a dor presente em doenças degenerativas podais que não responderam às terapias conservativas (Dabareiner et al., 1997; Zhang et al, 2006; Nicoletti et al., 2007; Koch, 2011; Rezaie; Mousavi; Mohajeri, 2011), são poucas as citações de neurectomia palmar (NP), principalmente em relação ao pós-cirúrgico e complicações inerentes em equinos. No caso descrito, a NP foi sugerida como forma de alívio permanente da dor crônica, visto o cloreto de amônio não apresentar a longevidade no tempo de ação necessária para o caso clínico relatado. A variação da técnica TG foi escolhida por ser mais simples e eficaz nas neurectomias digitais palmares segundo Faleiros et al.(2008); apresentarem menor proporção de tecido nervoso no tecido cicatricial em relação à TS, sugerindo menor favorecimento de regeneração nervosa (Escobar et al. 2008); e ocasionar menor índices de neuromas dolorosos em relação a TCE segundo Rezaie et al. (2011).

O resultado da NP foi satisfatório, ressaltando-se o aumento da dificuldade da técnica devido as aderências nervosas após a infiltração do neurolítico, durante aproximadamente 90 dias. Porém, mesmo com todos os cuidados em relação a TG e indicações pós-cirúrgicas, houve formação de neuroma doloroso, como resultado de uma regeneração desorganizada de axônios após lesão parcial ou completa. O neuroma torna-se doloroso quando este tecido desorganizado, frequentemente envolvido por tecido fibrocicatricial é estimulado por pressão, tensão e/ou hipóxia (Martins et al, 2002; Martins et al, 2015). Esses dados apresentados em nervos surais (Martins et al., 2002) e após amputação de dedos em humanos (Martins et al., 2015) trazem para reflexão o tempo de repouso mínimo necessário após a NP, podendo ser superior aos 45 a 60 dias descritos na média para NDP (Black, 1992; Dabareiner et al., 1997; Faleiros et 
al., 2008), além da cautela na utilização de ligas nas canelas de animais em competições, região da NP.

\section{CONCLUSÕES}

O cloreto de amônio a 2,0\% proporcionou analgesia de aproximadamente 40 dias na infiltração perineural em nervos palmares do caso relatado, sendo um neurolítico de médio tempo de ação (menor que 60 dias).

A NP foi eficaz em relação a analgesia e déficit de locomoção no animal relatado, porém após cerca de 110 dias apresentou como complicação pósoperatória neuromas dolorosos nos quatro sítios cirúrgicos. Há necessidade de mais relatos e pesquisas envolvendo a NP em equino avaliando: tempo e qualidade de analgesia; complicações após NP; riscos proprioceptivos ao animal; avaliações de fatores de risco na formação de neuromas após NP (pressão e modo de colocação de ligas de trabalho, manejo geral e treinamento).

\section{AGRADECIMENTOS}

Ao Conselho Nacional de Desenvolvimento Científico e Tecnólogico (CNPq) pelo financiamento de bolsas de produtividade e iniciação em desenvolvimento tecnológico e extensão inovadora.

\section{REFERÊNCIAS}

BLACK, J.B. Palmar digital neurectomy: an alternative surgical approach. Proc. Am. Assoc.Equine Pract., v.38, p.429-432, 1992.

DABAREINER, R. M.; WHITE, W. A.; SUlLINS, K. E. Comparsion of current techniques for palmar digital neurectomy in horses. Proc. Am. Assoc Equine Pract., v.43, p.231-232, 1997.

ESCOBAR, A.; RIO TINTO, J.J.M.; VASCONCELOS, A.C.; RACHID, M.A.; ALVES, G.E.S.; FALEIROS, R.R. Avaliação macroscópica, microscópica e histomorfométrica do tecido cicatricial pós cirúrgico de eqüinos submetidos a duas técnicas de neurectomia digital. Arq. Bras. Med. Vet. Zootec. V.60, n.4, p.800-805, 2008.

ESCODRO, P.B.; TONHOLO, J.; THOMASSIAN, A.; NASCIMENTO, T.G.; VILANI, R.G.D.C. Considerações acerca dos fármacos neurolíticos na medicina equina. Revista Brasileira de Medicina Equina, v.35, p.12-18, 2011.

ESCODRO, P.B.; SILVA, T.J.F.; MACEDO,J.S.; FONSECA, L.S; OLIVEIRA, C.F. et al.Infiltração de composição etanólica intra-articular associada à neurectomia palmar para tratamento de osteoartrite interfalângica proximal em equino: relato de caso. Jornal Brasileiro de Ciência Animal - JBCA, v.5, n.10, suplemento, p.238-240, 2012.

ESCODRO, P.B.; SILVA, T.J.F.; MARIZ, T.M.A.; GAIA, F.R.T.; TOLEDO, C.A.T.; BERNARDO, J.O. Uso de fármacos neurolíticos em equídeos de tração carroceiros: procedimento ilícito ou promoção de bem estar animal? Revista Brasileira de Medicina Equina, v. 60, p.4-9, 2015.
FALEIROS, R. R.; RIO TINTO, J. J.; ESCOBAR, A.; ALVES, G. E. S. Neurectomia digital em eqüinos: comparação das técnicas guilhotina e stripping. Arq. Bras. Med. Vet. Zootec., v.60, n.2, p.335-340, 2008.

GUASCO, P.G.; KELLY,G.; SCHUMACHER,J.; HENRY,R.W Excision of the Deep Branch of the Lateral Palmar Nerve of Horses to Resolve Lameness Caused by Proximal Suspensory Desmitis. Veterinary Surgery, v.42, p.296-301, 2013.

$\mathrm{KOCH}$ H. Painful neuroma - mid-term results of resection and nerve stump transposition into veins. Eur Surg, v.43, n.6, p. $378-381,2011$

MARTINS, R.S.; SIQUEIRA, M.G.; TEDESCO-MARCHESE, A.J. Neuroma sintomático do nervo sural. Arquivo Neuropsiquiatria, v.60, n.3-b, p.866-868, 2002.

MARTINS, R.S.; SIQUEIRA, M.G.; HEISE, C.O.; YENG, L.T.; ANDRADE, D.C.; TEIXEIRA, M.J. Interdigital direct neurorrhaphy for treatment of painful neuroma due to finger amputation. Acta Neurochir, v.157, p. 667-671, 2015.

NICOLETTI, J. L. M.; ESCODRO, P. B.; HUSSNI, C. A.; ALNES, A.L.; THOMASSIAN, A.; AMORIN, R.L. Estudo Comparativo experimental da injeção perineural do álcool benzílico $0,75 \%$ e do álcool etílico absoluto nos nervos palmares de equinos. Braz. J. vet. Res. anim. Sci, São Paulo, v. 44, n. 6, p. 401-407, 2007.

REZAIE,A.; MOUSAVI, G.; MOHAJER,D. A Comparative Histopathological Evaluation of Neuroma Formation Trough the Guilhotine and Epineural Capping Neurectomy Techniques in Horse. Global Veterinaria, v.6, n.5, p.498-451, 2011.

STASHAK, T.S. CLAUDICAÇÃO. In: STASHAK, T.S. (Ed). Claudicação em eqüinos segundo Adams. 4.ed. São Paulo: Roca, 1994. p.503-816. 